\title{
Effects of taste quality and intensity on oral perception of viscosity
}

\author{
CAROL M. CHRISTENSEN \\ Monell Chemical Senses Center, Philadelphia, Pennsylvania 19104
}

\begin{abstract}
Oral assessments of viscosity were obtained with the method of magnitude estimation. Subjects judged the viscosity of a series of aqueous solutions thickened to one of six viscosity levels (1-2025 centistokes) with a food-grade gum, sodium carboxymethylcellulose. The solutions contained one of several concentrations of caffeine, citric acid, sodium chloride, or sucrose. The presence of taste substances significantly altered the perception of solution viscosity for only the thickest solutions. Increasing concentrations of citric acid and sodium chloride produced progressive decreases in perceived viscosity, and increasing sucrose concentrations produced small increases in perceived viscosity. Caffeine did not affect judgments of solution viscosity. The most likely explanation for these findings is that subjects detected differences in the Newtonian behavior of the thickened solutions, differences that were produced by the addition of taste substances.
\end{abstract}

Humans can detect changes in solution viscosity using visual, nonoral, or oral somesthetic cues. Perceived changes in solution viscosity are compressed compared with concomitant changes in physical viscosity. The exponents of power functions relating physical and subjective measures of solution viscosity are .4 for visual and nonoral tactile determinations of viscosity (Stevens \& Guirao, 1964) and .3 for oral judgments of solution viscosity (Christensen, 1979). An exponent of .3 means that a 10 -fold increase in solution viscosity is perceived as only a 2 -fold increase in viscosity. It seems surprising that perception of viscosity is so similar when different sensory systems are utilized.

Addition of taste substances to viscous solutions could be expected to alter the oral perception of viscosity. Experiential factors might influence perception of solution viscosity; for example, thick beverages are more often sweet, whereas sour beverages are often thin. Such associations between taste and viscosity in certain foods could influence the perception of viscosity.

Based on neurophysiological considerations, the possibility also exists of oral texture-taste interactions. Oral tactile sensations conveyed by branches of the trigeminal nerve and gustatory sensations conveyed by branches of the facial and glossopharyngeal nerves project to adjacent portions of the major sensory areas in the central nervous system (Truex \& Carpenter, 1969), and there is some evidence that projections from both sensory systems synapse on some of the same neurons (Van Buskirk \& Erickson, 1977).

This research was supported in part by NIH Training Grant 5T32NS-07068 from NINCDS to the Monell Chemical Senses Center. The author expresses appreciation to the Hercules Corporation, Wilmington, Delaware, for providing the CMC, to Drs. Gary Beauchamp and Robert Cagan of the Monell Center for their helpful comments, and to Ms. Davis Diehl for her technical support.
The addition of certain taste substances could also lead to changes in perceived viscosity because they change solution viscosity or alter other physical properties related to the perception of viscosity. Research on the oral perception of viscosity reveals that humans appear to be sensitive to differences in the degree of Newtonian behavior of solutions (Christensen, 1979). The viscosity of a Newtonian solution does not vary with changes in its shear rate. Most foods, however, are non-Newtonian, which means that the apparent viscosity varies with shear rate. This usually means that viscosity decreases as shear rate increases. Humans perceive non-Newtonian solutions to be thinner than Newtonian solutions, suggesting that individuals take an "average" viscosity reading over the range of shear rates operating in the oral cavity.

Because there is reason to suspect viscosity-taste interactions, research was initiated to determine whether human perception of solution viscosity could be altered by adding taste substances. In this study, the effects of perceived viscosity were measured after adding three different concentrations of caffeine, citric acid, sodium chloride, or sucrose to solutions thickened to six different viscosity levels with a foodgrade gum, sodium carboxymethylcellulose.

\section{METHOD}

\section{Subjects}

Ten university students served as subjects. They were solicited by advertisements displayed on local campuses and were paid for their participation. Respondents were chosen in order of inquiry, with the restriction that equal numbers of females and males were chosen.

\section{Preparation of Test Stimuli}

Sodium carboxymethylcellulose, a largely odorless and tasteless gum (7MF, Hercules Corporation, Wilmington, Delaware), was slowly added to $150 \mathrm{ml}$ of an aqueous solution while the solution 
was being blended. The solutions were either deionized water alone or deionized water containing reagent grade $.01, .02$, or $.08 \mathrm{M}$ caffeine, $.012, .025$, or $.10 \mathrm{M}$ citric acid, $.10, .20$, or $.80 \mathrm{M}$ sodium chloride, or $.12, .25$, or $1.00 \mathrm{M}$ sucrose. Pilot studies were done to determine the amount of sodium carboxymethylcellulose (CMC) required to thicken each of the test solutions to $6,36,216,1,296$, and 2,025 centistokes (csk). In order to obtain a specific viscosity level, a different amount of $\mathrm{CMC}$ was required not only for every taste substance, but also for every concentration.

Blending speeds and times were carefully controlled and were different for the various solutions. A total of $900 \mathrm{ml}$ of each thickened solution was required for the study, and this quantity was obtained by repetitive preparation of $150-\mathrm{ml}$ batches of solution. Solutions were prepared at least $24 \mathrm{~h}$ in advance of viscosity measurements or their use in the study in order to allow for complete hydration of the polymer and stabilization of solution viscosity.

\section{Measurement of Physical Viscosity}

Cannon-Ubbelhode four-bulb shear dilution capillary viscometers (Models S122, S350, and S450; Cannon Instruments, State College, Pennsylvania) were used to determine solution viscosity. Viscosity is expressed as centistokes, but, because test solution densities were close to 1.00 , centistoke and centipoise values are very nearly comparable.

Viscosities were determined at shear rates of $100 \mathrm{sec}^{-1}$ for the 6-csk solutions, at $40 \mathrm{sec}^{-1}$ for the 36 -csk solutions, and at $10 \mathrm{sec}^{-1}$ for the $216-, 1,296-$, and 2,025 -csk solutions in order to comply with shear rates hypothesized to be operating in the oral cavity (Shama \& Sherman, 1973). Measurements were obtained at $27^{\circ} \mathrm{C}$, which was the highest temperature reached by room-temperature $\left(23^{\circ} \mathrm{C}\right)$ solutions held in the oral cavity for $5 \mathrm{sec}$.

After completing the sensory experiments, further measurements of some of the test solutions were made with a Wells-Brookfield microviscometer (Model RVT). This viscometer can measure solution viscosity over a much wider range of shear rates.

\section{Measurement of Perceived Viscosity}

The subjects used the method of magnitude estimation to report perceived viscosity (Stevens, 1975). The terms "viscosity" and "thickness" were equated in the instructions read to the subjects. Prior to judging solution viscosity, the subjects were given brief training in the use of magnitude estimation by being asked to judge the circumference of a series of circles.

The test samples were presented randomly to the subjects, but the first sample was an unflavored aqueous solution thickened to 216 csk. No numerical value was assigned by the experimenter to the first sample of the session (modulus-free design). The subjects were not permitted to use negative numbers or zero, but could use positive whole numbers, decimals, and fractions to express perceived viscosity. The subjects were instructed to give higher numbers to increasingly viscous solutions.

\section{Testing Procedures}

The subjects participated in four daily test sessions that each lasted approximately $1 \mathrm{~h}$. Only one taste quality was tested during a single session. The order of presentation of the taste qualities was randomized for each subject. Twenty-four different solutions were randomly presented twice to subjects in a given test session; aqueous solutions were either unthickened or thickened to one of five viscosity levels and contained either no taste substance or one of three concentrations of a taste substance.

The subjects sampled $10 \mathrm{ml}$ of each solution presented from a plastic, disposable syringe fitted with a $7.8-\mathrm{cm}$ piece of Tygon tubing which served as a straw. The Tygon tubing protruded through a slot in an opaque barrier that separated the experimenter and the subject. Efforts were made to hold constant the rate of solution delivery. Syringes were used for stimulus presentations in order to minimize nonoral cues and to control the volume of test solutions delivered.
The subjects were instructed to swish a solution around in their mouths as they would a mouthwash and then to judge solution viscosity before expectorating the solution. The subjects recorded their responses on individual slips of paper that were collected by the experimenter between solution presentations. Two deionized water rinses preceded the presentation of the next test solution.

\section{Analysis}

Before performing statistical analyses, the raw data were normalized for a single subject across the four test sessions using the unflavored aqueous series of thickened solutions that had been presented at every test session. Normalization between subjects was accomplished by obtaining differences between the appropriate $\log$ means. This difference score was then added to the individual scores (Engen, 1971). Appropriate power functions were calculated from the normalized data and t tests were used for post hoc comparisons of selected portions of the data (Winer, 1971). A .05 level represented statistical significance, but this value was adjusted for multiple comparisons by dividing it by the number of tests performed.

\section{RESULTS}

The addition of taste substances to viscous solutions significantly influenced judgments of solution viscosity. The most striking aspect of the data is the significant interactions between taste quality concentrations and physical viscosity levels. Figures 1 to 4 illustrate these relationships for each taste quality.

A three-factor analysis of variance (Taste Quality by Taste Concentration by Viscosity Level) demonstrated no main effect due to taste quality, indicating that the overall magnitude of perceived viscosity was not changed by taste quality. Consequently, twofactor analyses of variance were performed separately

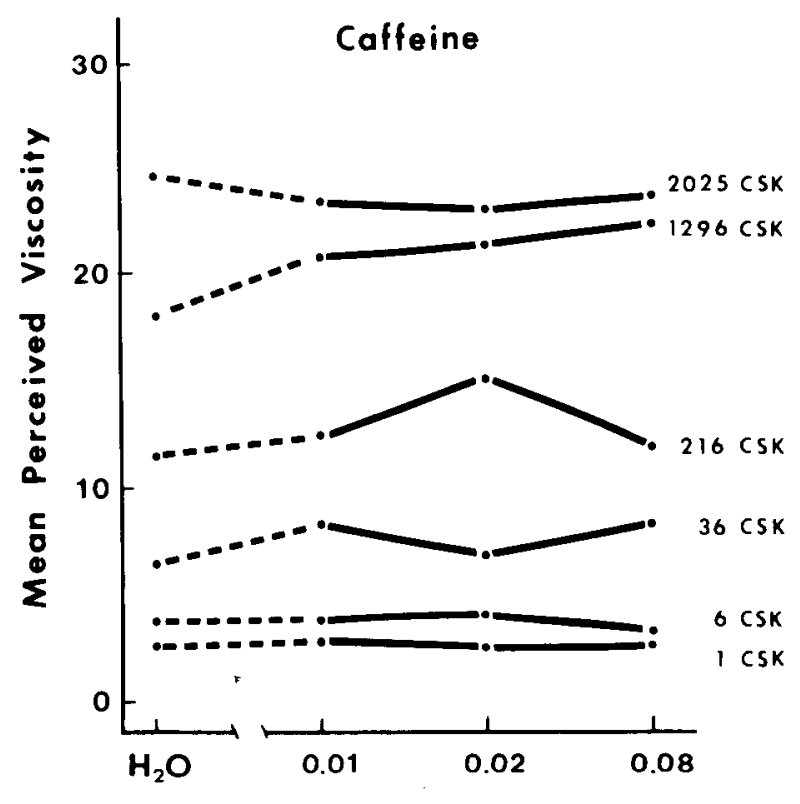

Molar Concentration

Figure 1. Effect of caffeine on perceived viscosity of solutions at six physical viscosity levels. Each point is the normalized mean for 10 subjects. 


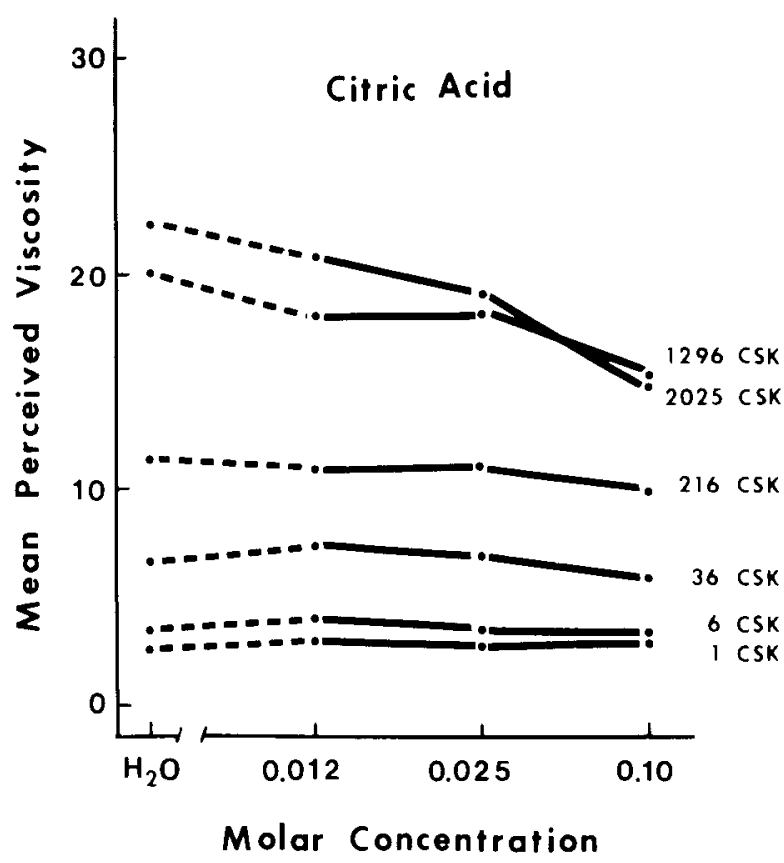

Figure 2. Effect of citric acid on perceived viscosity of solutions at six physical viscosity levels. Each point is the normalized mean for 10 subjects.

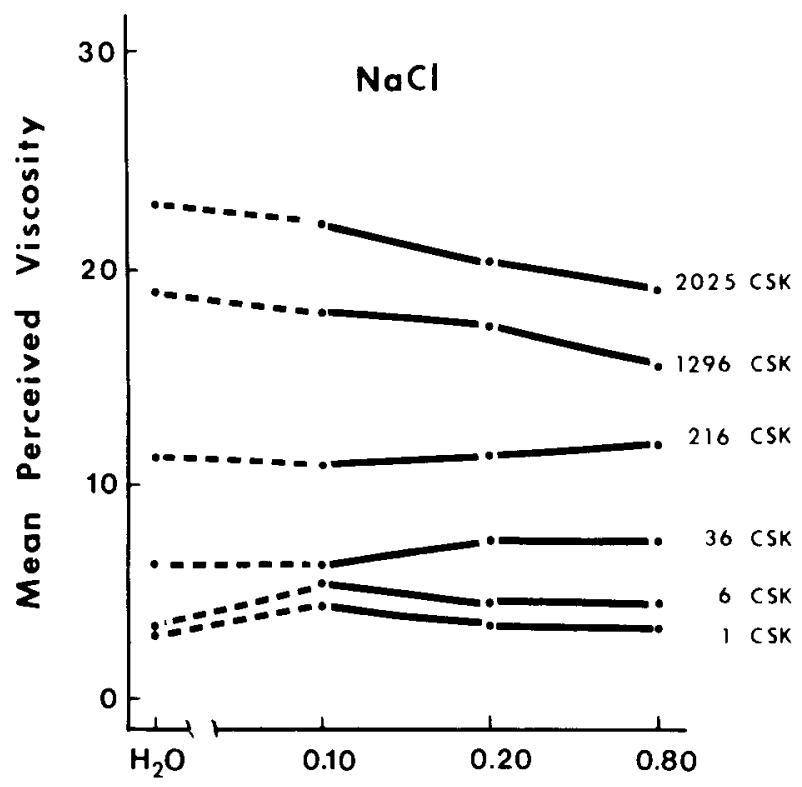

\section{Molar Concentration}

Figure 3. Effect of sodium chloride on perceived viscosity of solutions at six physical viscosity levels. Each point is the normalized mean for 10 subjects.

for each taste quality because of the difficulty in interpreting higher order interactions.

For each of the four taste qualities, there was a powerful main effect of viscosity level $(p<.001)$. With one exception (see Figure 2), increasingly vis- cous solutions were judged to be increasingly thick.

The main effect of taste concentration on perceived viscosity was significant only for citric acid $[\mathrm{F}(3,27)$ $=17.89, \mathrm{p}<.001]$. As illustrated in Figure 2, at each physical viscosity level, solutions containing higher concentrations of citric acid tended to be judged less thick than solutions with less citric acid.

Significant interactions between judgments obtained at different taste concentrations and levels of physical viscosity occurred with all four taste substances, but the pattern of interactions varied. Consequently, the results are described separately for each taste substance. In all cases, simple main effects were assessed at each viscosity level and judgments of pure aqueous solutions were compared with judgments of solutions containing the three taste concentrations.

Overall, it appears that the presence or absence of caffeine had no effect on perceived viscosity. There was a significant interaction between caffeine concentrations and viscosity level $[F(15,135)=2.28$, $\mathrm{p}<.01$ ], but there was no apparent pattern to these results. Post hoc tests revealed that the .02-M caffeine solution was judged to be significantly thicker than the unflavored 216-csk solution, and all three caffeine solutions were perceived to be thicker than the unflavored 1,296-csk solution.

The interaction was also significant for citric acid solutions $[\mathrm{F}(15,135)=3.81, \mathrm{p}<.001]$. As illustrated in Figure 2, subjects perceived solution viscosity to decrease with increasing citric acid concentrations, and this effect became more pronounced at progressively higher physical viscosity levels. At 1,296-csk, the .10-M citric acid solution was significantly less

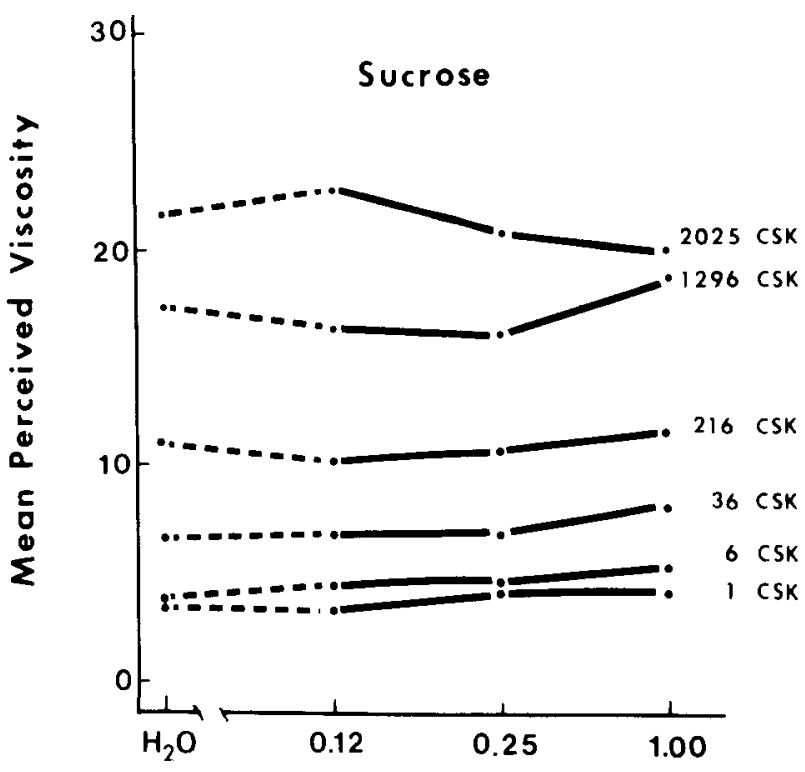

Molar Concentration

Figure 4. Effect of sucrose on perceived viscosity of solutions at six different physical viscosity levels. Each point is the normalized mean for 10 subjects. 
thick than the similarly thickened aqueous solution. At 2,025 csk, the .10- and .025-M citric acid solutions were perceived to be significantly less thick than the corresponding unflavored solution.

A similar pattern of interaction effects was observed with $\mathrm{NaCl}$ solutions $[\mathrm{F}(15,135)=1.90, \mathrm{p}<.05]$. At the highest viscosity levels, perceived viscosity decreased as $\mathrm{NaCl}$ concentration increased (see Figure 3). At both 1,296 and $2,025 \mathrm{csk}, .80-\mathrm{M}$ solutions were perceived to be thinner than aqueous solutions, and, at 2,025 csk, the .20-M solution was also significantly thinner than the aqueous solution.

A significant interaction was also found with the sucrose solutions $[F(15,135)=2.15, p<.05]$. As illustrated in Figure 4, at all but 2,025 csk, there was a weak trend of increases in perceived viscosity with increasing sucrose concentration. Post hoc tests did not reveal statistical differences for the selected comparisons.

The exponents of power functions relating physical and perceived viscosity for each taste concentration and quality range from .22 to $.30\left(\mathrm{r}^{2}\right.$ range: $\left..75-.85\right)$. The exponent of the same function for unflavored aqueous solutions is .29 . The range of exponents is $.22-.25$ for the three sucrose concentrations, $.23-.25$ for the $\mathrm{NaCl}$ concentrations, $.24-.28$ for the citric acid concentrations, and $.28-.30$ for the three caffeine concentrations. Figures 5 and 6 illustrate the relation-

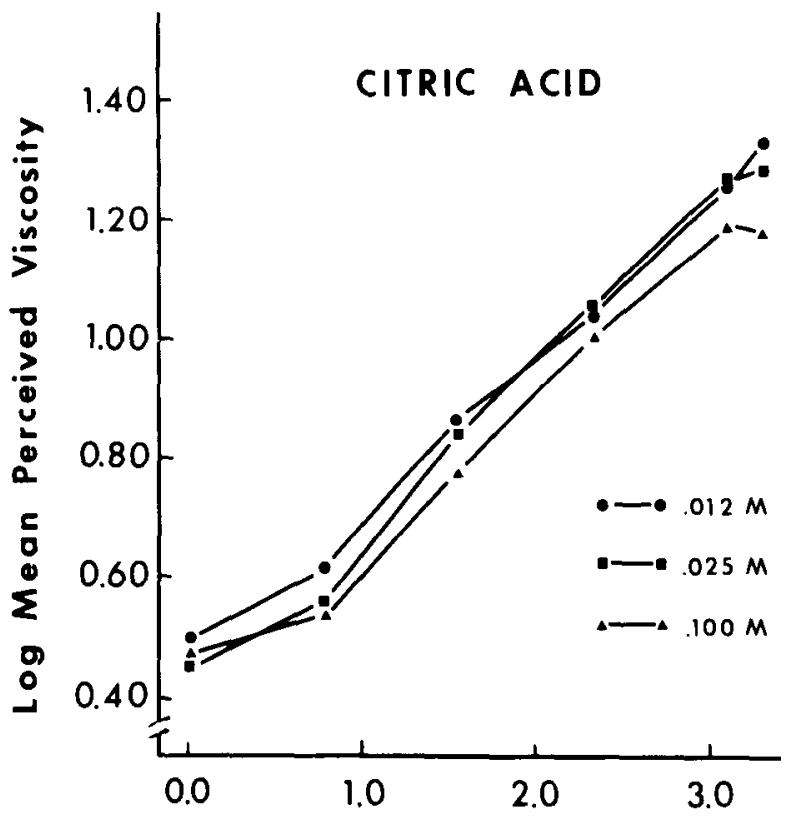

Log Viscosity (centistokes)

Figure 5. Relationship between log physical viscosity measured in centistokes and the log mean perceived magnitude of the viscosity of aqueous solutions containing different concentrations of citric acid. Each point is the normalized mean for 10 subjects.

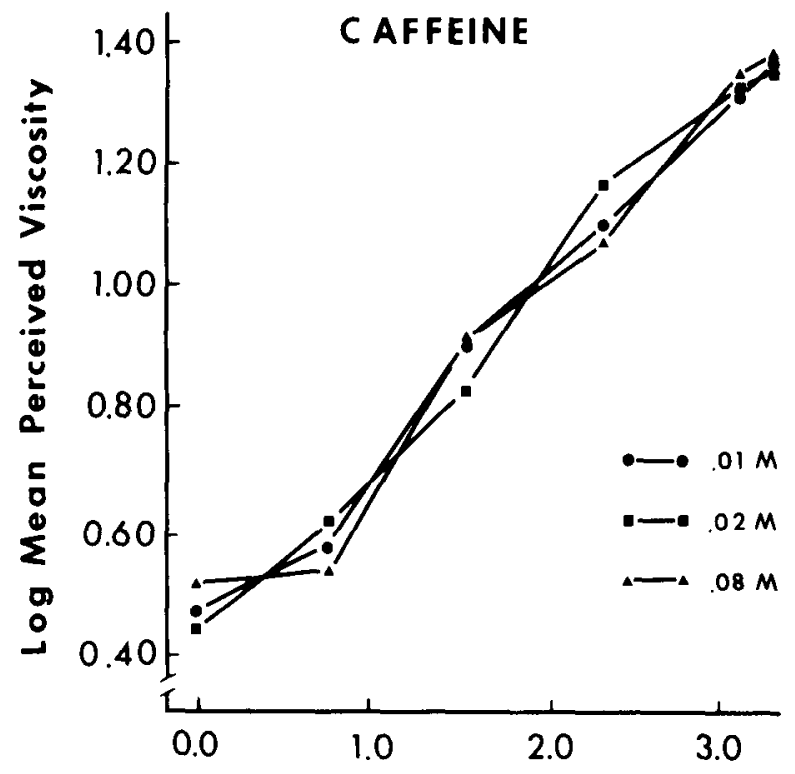

Log Viscosity (centistokes)

Figure 6. Relationship between log physical viscosity measured in centistokes and the $\log$ mean perceived magnitude of the viscosity of aqueous solutions containing different concentrations of caffeine. Each point is the normalized mean for $\mathbf{1 0}$ subjects.

ship between physical and perceived viscosity for thickened solutions of citric acid and caffeine.

After completion of the study, the low and high concentrations of caffeine, citric acid, sodium chloride, and sucrose were thickened to 6,216, and 2,025 csk with CMC-M. Solution viscosities were measured with a Wells-Brookfield microviscometer. The purpose of these measurements was to obtain viscosity values over a range of shear rates in order to examine the Newtonian character exhibited by the various solutions. Values for the 6-csk solutions were obtained from capillary viscometers.

The power functions, $\tau=x \cdot \gamma^{\eta}$, where $\tau=$ shear stress in dyn $\mathrm{cm}^{-2}$ and $\gamma=$ shear rate in $\mathrm{sec}^{-1}$ can be fitted to the physical viscosity data. The exponent expresses the degree of Newtonian behavior exhibited by a solution. A Newtonian solution yields an exponent of 1.00. Non-Newtonian solutions that thin as shear rate is increased produce exponents that are less than 1.00 .

Table 1 presents the exponents obtained from the series of thickened solutions. Two trends are apparent from the data: Thicker solutions are more nonNewtonian than thin solutions, and the addition of taste substances does alter a solution's Newtonian behavior, particularly for the thickest solutions. Increasing amounts of citric acid or sodium chloride increase the shear thinning of very thick solutions. Increasing concentrations of sucrose restore more 
Table 1

Power Function Exponent (n) Expressing Deviation from Newtonian Behavior

\begin{tabular}{llcc} 
& \multicolumn{3}{c}{ Viscosity (cps) } \\
\cline { 2 - 4 } \multicolumn{1}{c}{ Solution } & 6 & 216 & 2,025 \\
\hline $\mathrm{H}_{2} \mathrm{O}$ & .96 & .97 & .89 \\
$.10 \mathrm{M} \mathrm{NaCl}$ & .96 & .89 & .69 \\
$.80 \mathrm{M} \mathrm{NaCl}$ & .97 & .82 & .59 \\
$.012 \mathrm{M} \mathrm{Citric} \mathrm{Acid}$ & .95 & .88 & .74 \\
$.10 \mathrm{M} \mathrm{Citric} \mathrm{Acid}$ & .97 & .80 & .29 \\
$.125 \mathrm{M} \mathrm{Sucrose}$ & .97 & .94 & .77 \\
$1.00 \mathrm{M}$ Sucrose & .94 & .96 & .84 \\
$.01 \mathrm{M} \mathrm{Caffeine}$ & .96 & .93 & .79 \\
$.08 \mathrm{M}$ Caffeine & .99 & .92 & .79 \\
\hline
\end{tabular}

Note- $\gamma$ (shear stress) $=\gamma^{\eta}$ (shear rate) $\cdot x$ (consistency index). For 6-cps shear rate $=52-681 \mathrm{sec}^{-1} ;$ for 216-cps shear rate $=$ $4-77 \mathrm{sec}^{-1} ;$ for $2,025-\mathrm{cps}$ shear rate $=2-10 \mathrm{sec}^{-1}$.

Newtonian behavior. Caffeine has little effect on the Newtonian behavior of solutions, and there is no apparent concentration effect. Other research corroborates some of these findings of the effect of the addition of taste substances to CMC-thickened solutions (Ganz, 1977).

\section{DISCUSSION}

The addition of taste substances to viscous solutions alters the perception of solution thickness. Perceptual judgments of the thickest solutions $(1,296$ and 2,025 csk) were changed to the largest extent by the addition of taste substances. Increasing concentrations of citric acid and sodium chloride produced progressive decreases in the perceived viscosity of thick solutions. Increasing sucrose concentrations produced small rises in perceived solution viscosity. Caffeine had no systematic effect on viscosity judgments.

The most likely explanation for this set of results is that judgments of perceived viscosity were affected by alterations in the Newtonian behavior of the thickened solutions produced by the addition of taste substances. There is very good correspondence between the direction and degree of changes in perceived viscosity and the observed effects of caffeine, citric acid, sodium chloride, and sucrose on the shear-thinning properties of CMC-thickened solutions (see Table 1). Citric acid had the most pronounced effects on the Newtonian behavior of solutions and also produced the greatest changes in perceived viscosity. Increasing concentrations of either citric acid or $\mathrm{NaCl}$ led to decreases in the Newtonian character of the thickened solutions. Increasing amounts of sucrose produced small increases in Newtonian behavior, whereas the effect of caffeine was negligible.

Previous research demonstrated that individuals are sensitive to differences in the Newtonian character of solutions (Christensen, 1979). Subjects judged less Newtonian (shear-thinning) aqueous solutions to be thinner than more Newtonian aqueous solutions. The differences in Newtonian behavior were produced with different types of thickening agent, CMC. In the present study, changes in solution behavior were produced by taste substances. However, the results of the two studies are parallel: Newtonian solutions are perceived to be thicker than non-Newtonian solutions.

The test samples were prepared to have the same physical viscosity at shear rates hypothesized to be operating in the oral cavity (Shama \& Sherman, 1973). However, recent findings (Christensen, 1979) suggest that subjects sample solution viscosity over a wider range of shear rates than previously believed. As illustrated in Figure 7, the thicker samples in this study were prepared to have the same physical viscosity at $10 \mathrm{sec}^{-1}$ (see Procedures section). Completely Newtonian solutions (A) maintain the same viscosity regardless of shear rate. The CMC-M thickened aqueous solutions used in this study are nearly Newtonian. When a taste substance such as citric acid or $\mathrm{NaCl}$ is added, the solution behaves more like solution $\mathrm{B}$ or $\mathrm{C}$ in Figure 7; the CMC-thickened solutions exhibit shear-thinning. The low-molarity citric acid or $\mathrm{NaCl}$ solutions might resemble solution $\mathrm{B}$, and the higher concentrations would resemble solution $\mathrm{C}$. Subjects would most likely judge solution $\mathrm{C}$ to be thinner than $\mathrm{B}$ and would judge solution $\mathrm{A}$

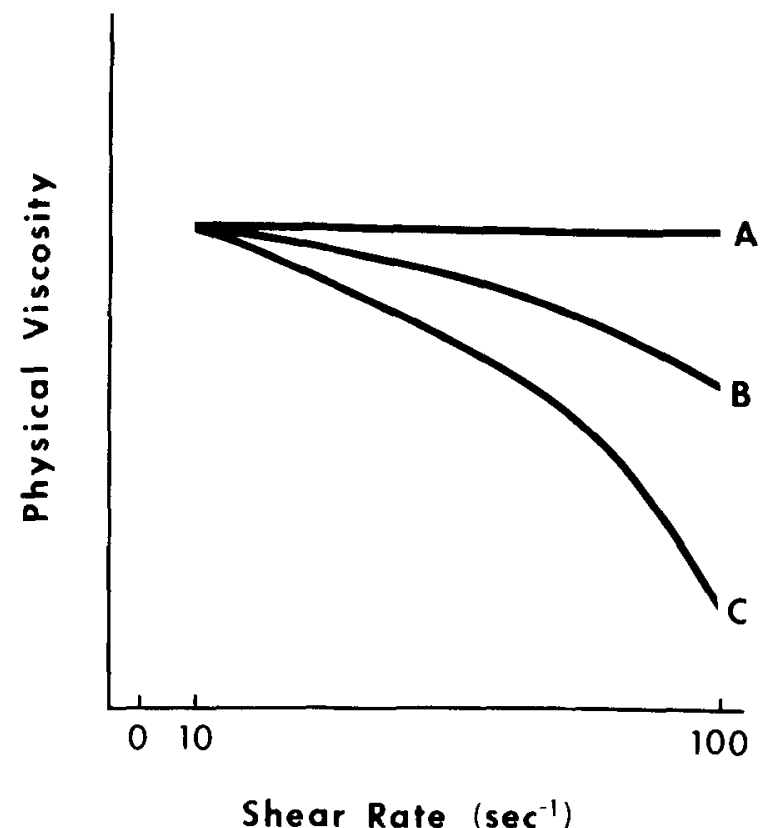

Figure 7. Illustration of the relationship between shear rate and physical viscosity for a Newtonian solution (A) and for solutions exhibiting different degrees of non-Newtonian, shear-thinning behavior (B) and (C). 
to be the thickest. A range of shear rates is expected to be generated in the oral cavity as a result of the method of stimulus delivery and the variable movements associated with swishing the solutions. The perception of solution viscosity may represent some form of average viscosity reading resulting from subjects' sampling varying solution viscosities along a physiologically relevant range of oral shear rates.

It is difficult to invoke either neural or learned associative mechanisms to account for the major finding of the study that judgments of only the most viscous solutions were affected by the presence of taste substances. The possibility that "crosstalk" between the neural systems serving the oral tactile and gustatory sensations contributed to the results does not seem likely. Such a model might predict either that there would be a similar effect of a particular taste quality on perceived viscosity regardless of viscosity level or that judgments of the thin solutions (weakest signal) would be most affected by the addition of taste substances.

Experiential factors may have contributed to the observed results, since increasingly sour solutions were perceived as less thick and increasingly sweet solutions were perceived to be thicker. Again, however, it is difficult to use a learning model to account for the thickest solutions differentially being most affected by the presence of taste substances.

The results of this study add to the growing evidence for oral texture-taste interactions. The site of interaction appears to be the oral cavity when solution viscosity and taste additives are manipulated. Taste substances can alter certain physical characteristics of thickened aqueous solutions, and individuals appear able to detect these textural changes.

\section{REFERENCES}

Christensen, C. M. Oral perception of solution viscosity. Journal of Texture Studies, 1979, 10, 153-164.

ENGEN, T. Psychophysics: II. Scaling methods. In J. W. Kling \& L. A. Riggs (Eds.), Woodworth and Schlosberg's experimental psychology. New York: Holt, Rinehart \& Winston, 1971.

Ganz, A. J. Cellulose hydrocolloids. In H. D. Graham (Ed.), Food colloids. Westport, Conn: AVI, 1977.

Shama, F., \& Sherman, P. Identification of stimuli controlling the sensory evaluation of viscosity: II. Oral methods. Journal of Texture Studies, 1973, 4, 111-118.

Stevens, S. S. Psychophysics (G. Stevens, ed.). New York: Wiley, 1975.

Stevens, S. S., \& Guirao, M. Scaling of apparent viscosity. Science, 1964, 144, 1157-1158.

Truex, R. C., \& Carpenter, M. B. Human neuroanatomy. Baltimore: Williams and Wilkins, 1969.

Van Buskirk, R. L., \& Erickson, R. P. Odorant responses in taste neurons of the rat NTS. Brain Research, 1977, 135, 287-303.

Winer, B. J. Stutistical principles in experimental design. New York: McGraw-Hill, 1971.

(Received for publication March 12, 1980; revision accepted July 16,1980 .) 